\title{
Novel Pravastatin-Producing Penicillium janthinellum Strain Isolated from Soil
}

\author{
Emine Seydametova ${ }^{1,2^{*}}$ \\ 1 Faculty of Chemical and Natural Resources Engineering, Universiti Malaysia Pahang, Kuantan, Pahang State \\ Malaysia. \\ 2 Institute of Microbiology, Academy of Sciences of Uzbekistan, Tashkent, Uzbekistan. \\ * Corresponding author. Tel.: +6010-9093806; email: dr.seydametova@gmail.com \\ Manuscript submitted February 6, 2015; accepted March 31, 2015. \\ doi: 10.17706/ijbbb.2015.5.2.80-90
}

\begin{abstract}
Hyperlipidemia is a main risk factor for coronary heart disease and stroke. The lipid-lowering drug, pravastatin, is currently obtained by the microbial biotransformation of compactin (mevastatin) to pravastatin. It is clear that pharmaceutical industry has to date utilized only a very minor portion of nature's microbial arsenal for the discovery of pravastatin. Therefore, the search for microfungi which is able to produce this statin directly has gained increasing importance. In this study, a total of 33 soil fungal cultures were isolated from nine sampling sites in oil palm plantations in Malaysia and tested for their potential to produce pravastatin. The cultures were cultivated in submerged fermentation followed by screening for statin production using high-performance liquid chromatography. The best pravastatin producer was identified to species level using morphological characteristics and molecular biological approaches. From the tested cultures, 18 fungal isolates were able to produce pravastatin directly by fermentation. Among these, the isolate ESF20P was the best producer, with a yield of $15.8 \mathrm{mg} / \mathrm{l}$ pravastatin. Molecular identification of this strain showed the highest homology (98\%) with Penicillium janthinellum.
\end{abstract}

Key words: Microfungi, pravastatin, screening, soil.

\section{Introduction}

Cholesterol is an essential lipid component of the human cell membrane and a precursor for steroid hormones and bile acids. However, any biomolecule in excess can be harmful for human health. Because lipids are carried in the bloodstream, hyperlipidemia is always a threat to coronary arteries and the most important risk factor for coronary heart disease and stroke [1]. To fight these problems, medical science has increasingly relied on several drugs, commonly known as lipid-lowering drugs. One group of these drugs known as statins lowers cholesterol by interfering with the cholesterol biosynthetic pathway [1], [2]. Only one-third of total body cholesterol is diet-derived; while two-thirds are synthesized by the liver and, to a lesser extent, by other organs [3], [4]. For this reason, control of cholesterol by inhibiting its biosynthesis provides an important strategy to lower cholesterol levels in the blood.

Statins selectively inhibit 3-hydroxy-3-methylglutaryl-coenzyme A (HMG-CoA) reductase, the regulatory and rate-limiting enzyme in cholesterol biosynthesis. The remarkable safety of these drugs derives from their unique mechanisms of action. The inhibition of HMG-CoA reductase results in accumulation of HMG-CoA, which can be metabolized to simpler compounds, without any buildup of lipophilic intermediates with a sterol ring [5], [6]. In this way, statins lower cholesterol, thus, preventing plaque 
buildup within the arteries.

There are currently several statins commercially available in pharmaceutical form [1], [7]. In comparison with other statins, pravastatin is a highly selective natural inhibitor of hepatic cholesterol synthesis. It is taken specifically into hepatic cells via a carrier-mediated active transport system and inhibits cholesterol synthesis selectively in the liver [8]. This lipid-lowering agent has been studied with reference to its long-term lipid-lowering effect, patient tolerance and clinical safety [9], [10]. In both studies, no serious clinical or laboratory abnormalities were observed, supporting the fact that pravastatin seems to the HMG-CoA reductase inhibitor that is best tolerated in comparison with other natural, semi-synthetic and synthetic statins.

Pravastatin currently can be obtained on an industrial scale only by the biotransformation of compactin (mevastatin) produced by Penicillium strains [7]. However, the conversion of compactin to pravastatin is itself inhibited by its own high concentration. This apparently is an unavoidable limitation of the microbial biotransformation process. The development of a one-step fermentation process for production of pravastatin based on an active microbial producer could therefore provide a novel and effective alternative. Therefore, in current study, the isolation of soil microfungi and assessment of their ability to produce pravastatin directly were required.

\section{Materials and Methods}

\subsection{Soil Sampling}

Soil samples for microfungal isolation were obtained from oil palm plantations of different ages located in Gambang (Yayasan Pahang Plantation Holding Sdn Bhd, Gambang, Pahang State, Malaysia). Thirty soil samples were randomly collected from 10 sampling sites. Samples were returned to the laboratory rapidly for processing. These soil samples were used to determine soil pH in the laboratory [11].

\subsection{Isolation of Microfungi}

Microfungi were isolated using the soil plate method of Warcup [12]. A small amount of soil (5-15 mg) was dispersed in liquid $\left(40^{\circ} \mathrm{C}\right)$ PDA medium in a sterile Petri dish without prior suspension in water. Control and experimental plates were prepared in triplicate. Inoculated Petri dishes were incubated at $25^{\circ} \mathrm{C}$ until the colonies were fully formed. No growth was observed on control plates containing PDA medium alone. Individual fungal colonies were picked and purified by streaking onto fresh PDA medium. After several cycles of picking and replating, separate colonies were transferred to PDA slants.

\subsection{Fermentation Procedure}

The fungal strains were maintained on PDA slants and incubated at $25^{\circ} \mathrm{C}$ for 7 days. A spore suspension was prepared by suspending spores from the appropriate slant in $10 \mathrm{ml}$ of sterilized distilled water containing $0.01 \%(\mathrm{v} / \mathrm{v})$ Tween-80. The number of spores was counted using a haemocytometer after vortexing and appropriate dilution. Ten percent $(\mathrm{v} / \mathrm{v})$ of the medium volume of the spore suspension $\left(10^{8}\right.$ spores $/ \mathrm{ml}$ ) prepared from the slant was used to inoculate sterile screening medium containing: $3 \%(\mathrm{w} / \mathrm{v}$ ) glucose, $3 \%\left(\mathrm{w} / \mathrm{v}\right.$ ) glycerol, $0.4 \%(\mathrm{w} / \mathrm{v})$ peptone, $0.2 \%(\mathrm{w} / \mathrm{v}) \mathrm{NaNO}_{3}, 0.1 \%(\mathrm{w} / \mathrm{v}) \mathrm{MgSO}_{4} \cdot 7 \mathrm{H}_{2} \mathrm{O}$. The pH value of the medium was adjusted to 6.5 with either $10 \% \mathrm{HCl}$ or $10 \% \mathrm{NaOH}$ before sterilization [13]. Shake flask fermentation was carried out in a 100 -ml flask at $25^{\circ} \mathrm{C}, 180 \mathrm{rpm}$, for 7 days in an incubator shaker (Multitron Version 2; Infors AG, Switzerland).

\subsection{Analytical Determination of Statins}

Thirty-three fungal isolates obtained from the soil samples were tested for their ability to produce mevastatin (precursor of pravastatin) and pravastatin. Ethyl acetate extracts from the whole cell broths 
obtained according to the slightly modified extraction procedure of Manzoni et al. [14] were used for HPLC analysis (Agilent 1200; Agilent Technologies, USA) of mevastatin and pravastatin. The $\mathrm{pH}$ value of the whole cell broth was adjusted to $3 \pm 0.2$ with $1 \mathrm{M}$ trifluoroacetic acid, and then an equal volume of ethyl acetate was added. Extraction was performed in an Ecotron incubator shaker (Infors HT, Switzerland) at $200 \mathrm{rpm}$ and $30^{\circ} \mathrm{C}$ for $1 \mathrm{~h}$. The fermentation samples were subsequently filtered through a Whatman filter paper no. 41 and the organic phase of each sample was collected using a nonsterile hydrophobic PTFE syringe filter (pore size: $0.22 \mu \mathrm{m}$ ). The ethyl acetate filtrates were then dried over anhydrous $\mathrm{Na}_{2} \mathrm{SO}_{4}$ followed again by filtration from the drying agent through a nonsterile hydrophobic PTFE syringe filter (pore size: $0.22 \mu \mathrm{m}$ ), and concentrated using a rotary evaporator to a final volume of $4 \mathrm{ml}$. Five microliters from the organic phase were then injected for HPLC analysis on a $250 \times 4.6 \mathrm{~mm}$ ID Zorbax Eclipse Plus $\mathrm{C}_{18}$ column, $5 \mu \mathrm{m}$ particle size (Agilent Technologies, USA). The mobile phase consisted of acetonitrile and water $(60: 40, \mathrm{v} / \mathrm{v})$ with a $\mathrm{pH}$ value adjusted to $\mathrm{pH} 3 \pm 0.2$ by addition of $1 \mathrm{M} \mathrm{H}_{3} \mathrm{PO}_{4}$ [13], [14]. The flow rate was maintained at $0.8 \mathrm{ml} / \mathrm{min}$ and detection was measured at $238 \mathrm{~nm}$. HPLC grade mevastatin ( $\geq 95 \%$ purity) and pravastatin ( $\geq 98 \%$ purity) from Sigma-Aldrich (USA) were used as standards.

\subsection{Dry Cell Weight Estimation}

Dry cell weight (DCW) was measured by filtering the contents of each flask through a pre-weighed Whatman filter paper no. 41 . The collected biomass was washed with distilled water and dried at $105^{\circ} \mathrm{C}$ in a UT6 model laboratory oven (Heraeus Instruments, Germany) until constant weight.

\subsection{Cultural and Morphological Studies of the Selected Strain}

The fungal strain selected in this study was transferred to appropriate diagnostic agar media for identification [15], [16]: Czapek yeast autolysate (CYA) agar and malt extract agar (MEA). Culture was incubated in the dark at $25^{\circ} \mathrm{C}$ for 7 days. Fungal isolate was identified morphologically following Matsukuma et al. [16]. The macroscopic features examined included colony diameter, obverse and reverse colony colors. The standard medium used for microscopic observations was MEA. Conidiophore branching patterns were examined using an optical microscope (Primo Star Carl Zeiss, Germany). These techniques allowed the fungal isolate to be identified at the species level.

\subsection{Molecular Identification of the Selected Strain}

The strain was maintained on a PDA slant. The pure isolate was cultured in a 100 -ml conical flask containing $20 \mathrm{ml}$ of CYA liquid medium. The culture was inoculated with a spore suspension and incubated at $25^{\circ} \mathrm{C}$ with shaking $(150 \mathrm{rpm})$ in an Ecotron microbiological incubator (Infors HT, Switzerland). Mycelial biomass from 2-day-old culture was harvested by filtration through Whatman filter paper no. 1 and used for DNA isolation.

Genomic DNA was obtained using the Fungi/Yeast Genomic DNA Isolation Kit (Norgen Biotek Corporation, Canada), according to the slightly modified manufacturer's instructions. Fifty milligrams of fungus (wet mass) were transferred to $1 \mathrm{ml}$ of $0.85 \% \mathrm{NaCl}$ in a microcentrifuge tube and centrifuged at 13,000 rpm for $1 \mathrm{~min}$ in a Biofuge Pico microcentrifuge (Heraeus Instruments, Germany) to pellet the cells. The supernatant was poured off carefully and $500 \mu \mathrm{l}$ of lysis solution was added to the cell pellet, and the cells were re-suspended by gentle vortexing using a Vortex Mixer Classic (VELP Scientifica, Italy). The mixture was transferred to the provided bead tube and vortexed for $5 \mathrm{~min}$ at $400 \mathrm{rpm}$ in an Ecotron microbiological incubator (Infors HT, Switzerland). The bead tube with lysate was then incubated at $65^{\circ} \mathrm{C}$ for $10 \mathrm{~min}$ in a water bath (Memmert, Germany). All of the lysate, including cell debris but not beads, was transferred to a DNA-free microcentrifuge tube by pipetting and was centrifuged for 2 min at 13,000 rpm. Clean supernatant was carefully transferred to a new DNA-free microcentrifuge tube, and the volume was 
noted. An equal volume of $70 \%$ ethanol was added to the collected lysate and vortexed to mix, after which $600 \mu \mathrm{l}$ of the lysate with ethanol was applied onto the spin column with collection tube and centrifuged for $1 \mathrm{~min}$ at 13,000 rpm. Then, $500 \mu \mathrm{l}$ of wash solution was applied to the column and centrifuged for $1 \mathrm{~min}$. This column washing step was repeated once more. The column was placed into a fresh 1.7-ml elution tube provided with the kit and $100 \mu \mathrm{l}$ of elution buffer was added to the column. The mixture was centrifuged for $2 \mathrm{~min}$ at 2,000 rpm, followed by $1 \mathrm{~min}$ at 13,000 rpm. The concentration of DNA diluted 25 times by mixing $1 \mu \mathrm{l}$ of sample with $24 \mu \mathrm{l}$ of molecular grade water was determined at $260 \mathrm{~nm}$ using Eppendorf Biophotometer Plus (Eppendorf AG, Germany). Then, the final concentration of DNA was adjusted to 2.5 $\mu \mathrm{g} / \mathrm{ml}$ by preparation of an appropriate dilution of a stock DNA solution. The DNA purity was checked using Eppendorf BioPhotometer Plus (Eppendorf AG, Germany) (OD 260/280 nm), and the DNA integrity was checked by electrophoresis at $70 \mathrm{~V}$ for $30 \mathrm{~min}$ on $1 \%$ agarose gel in a Sub-Cell GT agarose gel electrophoresis system (Bio-Rad Laboratories, USA). The purified genomic DNA was stored at $-20^{\circ} \mathrm{C}$ in a laboratory freezer (Revco, USA) until required.

The ITS1-5.8S-ITS2 region of the nuclear rDNA from the isolate was amplified using forward primer ITS1 (5'-TCCGTAGGTGAACCTGCGG-3') and reverse primer ITS4 (5'-TCCTCCGCTTATTGATATG-3') [17], obtained from Vivantis Technologies, USA. These primers are considered as universal fungal primers. The amplification reaction was carried out in volume of $25 \mu \mathrm{l}$ containing $4 \mu \mathrm{l}(10 \mathrm{ng})$ of template DNA, $1.5 \mu \mathrm{l}$ of each primer $(20 \mu \mathrm{M}), 2.5 \mu \mathrm{l}$ of 10×PCR buffer, $1 \mu \mathrm{l}$ of $\mathrm{MgCl}_{2}(50 \mathrm{mM}), 0.25 \mu \mathrm{l}$ of dNTPs $(100 \mathrm{mM})$ and $0.25 \mu \mathrm{l}$ of Taq DNA polymerase ( $5 \mathrm{U} / \mu \mathrm{l}$ ) supplied by Vivantis Technologies, USA [18]. A negative control, containing all reagents except for genomic DNA, was also prepared. The PCR reaction was performed in an Eppendorf Mastercycler Vapoprotect (Eppendorf AG, Germany) using $10 \mathrm{ng}$ of genomic DNA measured with Eppendorf Biophotometer Plus (Eppendorf AG, Germany). The amplification program used was described by Henry et al. [19] and included one cycle of $4 \mathrm{~min} 30 \mathrm{~s}$ at $95^{\circ} \mathrm{C}$ (predenaturing), 40 cycles of $30 \mathrm{~s}$ at $95^{\circ} \mathrm{C}$ (denaturation), $30 \mathrm{~s}$ at $50^{\circ} \mathrm{C}$ (annealing), $60 \mathrm{~s}$ at $72^{\circ} \mathrm{C}$ (extension) and finally one cycle of $3 \mathrm{~min}$ at $72^{\circ} \mathrm{C}$. For PCR, $5 \mu$ of PCR product was examined by electrophoresis at $90 \mathrm{~V}$ for $1 \mathrm{~h} 50 \mathrm{~min}$ in a $1 \%(\mathrm{w} / \mathrm{v})$ agarose gel stained with GoodView nucleic acid stain (Beijing SBS Genetech Co. Ltd., China) in 1×TAE buffer in a Sub-Cell GT agarose gel electrophoresis system (Bio-Rad Laboratories, USA). The molecular mass of the amplified DNA was estimated by comparison with a ready-to-use VC 100-bp Plus DNA ladder (Vivantis Technologies, USA) as a molecular size marker. The agarose gel was visualized under UV light using an Alpha Ease FC Imaging system (Alpha Innotech, Germany).

The obtained PCR product was sequenced by First Base Laboratories Sdn Bhd, Malaysia. The PCR fragment was extracted from the agarose gel and purified with GF-1 AmbiClean Kit (Vivantis Technologies, USA) following the manufacturer's instructions. The purified PCR fragment was sequenced on both strands using forward (ITS1) or reverse (ITS4) primers. Genetic sequencing of the amplified ribosomal sequences was carried out utilizing the BigDye Terminator v3.1 Cycle Sequencing Kit (Applied Biosystems, USA). Following removal of dye terminators using BigDye XTerminator Purification Kit (Applied Biosystems, USA), samples were analyzed on an ABI PRISM 3730xL Genetic Analyzer (Applied Biosystems, USA), utilizing Sequence Scanner software version 1.0.

The sequences obtained were pairwise aligned using the SDSC Biology Workbench 3.2 software [20] with default settings. For the identification of the fungal isolate, percentage of sequence identity and coverage were compared with available sequences in GenBank using the Basic Local Alignment Tool, BLASTN program (http://blast.ncbi.nlm.nih.gov/Blast.cgi).

\subsection{Phylogenetic Analysis}

Phylogenetic analysis was performed from aligned sequences of the rDNA ITS region containing ITS1 and ITS2 and the intervening 5.8S rRNA gene. The sequences were aligned using ClustalW with all multiple 
alignment parameters used at default settings. The sequence alignment included Penicillium janthinellum ESF20P (JX456373), Penicillium janthinellum CBS 340.48 (GU981585), Penicillium citrinum CBS 122452 (GU944576), Penicillium janczewskii CBS 354.48 (KC411755), Penicillium brevicompactum CBS 257.29 (KF465776), Penicillium bialowiezense CBS 227.28 (EU587315), Penicillium chrysogenum CBS 306.48 (JX997093), Penicillium flavigenum CBS 419.89 (JX997105), Penicillium viridicatum CBS 390.48 (JN942697), Penicillium cyclopium CBS 144.45 (JN942742), Penicillium aurantiogriseum CBS 324.89 (JN942751), Penicillium roqueforti CBS 221.30 (KF465778) and Penicillium paneum CBS 465.95 (HQ442349) species available from GenBank. Talaromyces bacillisporus CBS 296.48 (JN899329) was the outgroup species. Cladistic analysis using neighbour-joining method was performed with the MEGA 4.0 computer program [21]. Phylogenetic distances were calculated using Jukes-Cantor model. Confidence values for individual branches were determined by 1000 bootstrap replications.

\subsection{Statistical Analysis}

Each experiment was conducted in three replicates. Mean values with standard deviation were presented.

\section{Results and Discussion}

\subsection{Fungal Isolates Recovered from Oil Palm Plantation Soils}

Microfungi are one of the most common microorganisms in both cultivated and uncultivated soils, and have been isolated from numerous soils worldwide. Some fungal genera have representative species in most soils. For example, the genera Aspergillus, Penicillium, Fusarium and Trichoderma occur very frequently [22]. Although microfungi are commonly distributed in soils, our knowledge of fungal diversity in tropical ecosystems is limited [23]. The present study was carried out using cultivated soils from oil palm plantations. As soils from these habitats contain significant amounts of lipid compounds [11], microfungi isolated from these soils may produce antihypercholesterolemic agents such as pravastatin. In this study, 30 soil samples from 10 sampling sites in oil palm plantations were examined (Table 1).

Table 1. Soil Samples Obtained from Oil Palm Plantations in Gambang (3.72 $\left.{ }^{\circ} \mathrm{N} 103.12^{\circ}-\mathrm{E}\right)$

\begin{tabular}{lll}
\hline \hline Sampling site & Age of oil palm plantation & Sampling depth $(\mathrm{cm})$ \\
\hline A & 10-year-old & 10 \\
B & 10-year-old & 20 \\
C & 10-year-old & 30 \\
D & 10-year-old & 40 \\
E & 10-year-old & 50 \\
F & 15-year-old & 10 \\
G & 15-year-old & 20 \\
H & 15-year-old & 30 \\
I & 20-year-old & 10 \\
J & 20-year-old & 20 \\
\hline \hline
\end{tabular}

A total of 33 fungal isolates were recovered from nine sampling sites, with all of the source soils showing acidic conditions (pH ranging from 3.15 to 5.29) favourable for the growth of microfungi. No fungal isolates were recovered from site $\mathrm{F}$. The majority of the isolates (22 out of 33) were recovered from the sandy soils of a 10-year-old oil palm plantation characterized by moderate moisture content and $\mathrm{pH}$ ranging from 4.40 to 5.29. In total nine isolates were recovered from the peat soils of a 15-year-old plantation, while only two isolates were recovered from the deep peat soils of a 20 -year-old plantation. 


\subsection{Statin-Producing Ability of Fungal Isolates}

Previous studies have utilized different methods for the screening of natural statins by microfungi. Thin-layer chromatography (TLC) has been used to detect mevinolin (lovastatin) production [24]. Kumar et al. [25] developed a more economical and less labor-intensive 'agar plug' method, utilizing the anti-fungal property of lovastatin to produce a zone of inhibition against Neurospora crassa. Chakravarti and Sahai [26] described a spectrophotometric method for the determination of mevastatin (compactin), the pravastatin precursor. In the present study, to investigate the ability of the fungal isolates obtained to produce mevastatin and pravastatin, samples were examined using HPLC. To increase the sensitivity of the analytical method, ethyl acetate was used to extract these statins from the whole cell broths.

Several fungal genera have been reported to be able to produce mevinolin (lovastatin) directly by fermentation [14], [24], [27]. Among the few microbial strains for mevastatin production are Penicillium citrinum [26], Penicillium cyclopium [28], and Penicillium brevicompactum [29]. Although mevastatin is not used directly in treatment, it is an important intermediate compound in industrial pravastatin production [7].

In current study, eighteen of the 33 cultures obtained produced pravastatin at different levels (Table 2). Of these, the isolate ESF20P was the best producer, achieving a concentration of $15.80 \mathrm{mg} / \mathrm{l}$ pravastatin, equating to $1096.72 \mu \mathrm{g} / \mathrm{g}$ dry cell weight. The standard pravastatin ( $\geq 98 \%$ purity) used showed a peak with $R_{t}$ of 3.216 min (Fig. 1(a)) in current study, while that extracted from the isolate ESF20P showed a peak of $3.205 \mathrm{~min}$ (Fig. 1(b)), both also very similar to the $R_{t}(3.19 \mathrm{~min}$ ) reported by Manzoni et al. [14]. The next most active pravastatin producers were the isolates ESF26P and ESF32P, with $13.89 \mathrm{mg} / \mathrm{l}$ and 10.85 $\mathrm{mg} / \mathrm{l}$ pravastatin, respectively.

Table 2. Pravastatin-Producing Fungal Isolates

\begin{tabular}{cll}
\hline \hline Isolate & Pravastatin $(\mathrm{mg} / \mathrm{l})$ & Pravastatin $(\mu \mathrm{g} / \mathrm{g}$ DCW $)$ \\
\hline ESF6P & $1.07 \pm 0.11$ & $67.74 \pm 7.12$ \\
ESF11P & $1.23 \pm 0.06$ & $103.96 \pm 11.95$ \\
ESF16P & $1.02 \pm 0.17$ & $36.91 \pm 2.95$ \\
ESF18P & $1.27 \pm 0.12$ & $105.53 \pm 9.95$ \\
ESF19P & $1.39 \pm 0.06$ & $74.64 \pm 7.24$ \\
ESF20P & $15.80 \pm 1.90$ & $1096.72 \pm 84.36$ \\
ESF23P & $4.70 \pm 0.57$ & $347.21 \pm 21.87$ \\
ESF24P & $3.85 \pm 0.65$ & $351.41 \pm 24.93$ \\
ESF25P & $0.87 \pm 0.18$ & $81.74 \pm 8.80$ \\
ESF26P & $13.89 \pm 1.54$ & $959.27 \pm 70.48$ \\
ESF27P & $6.44 \pm 0.87$ & $594.58 \pm 49.02$ \\
ESF28P & $9.24 \pm 1.12$ & $927.11 \pm 88.81$ \\
ESF29P & $2.36 \pm 0.36$ & $356.47 \pm 33.32$ \\
ESF30P & $7.27 \pm 0.86$ & $554.58 \pm 32.89$ \\
ESF31P & $7.96 \pm 0.69$ & $1149.28 \pm 83.39$ \\
ESF32P & $10.85 \pm 1.23$ & $738.41 \pm 75.09$ \\
ESF33P & $4.13 \pm 0.49$ & $322.03 \pm 31.20$ \\
ESF34P & $3.82 \pm 0.61$ & $374.20 \pm 32.04$ \\
\hline
\end{tabular}

aResults are presented as mean \pm standard deviation from three replicates. bDCW=dry cell weight.

All 33 isolates were also screened for mevastatin production ( $R t$ of $14.602 \mathrm{~min}$ ), with no traces of this compound being found. The results obtained here suggest that pravastatin-producing strains immediately transformed any mevastatin produced into pravastatin. 
(a)

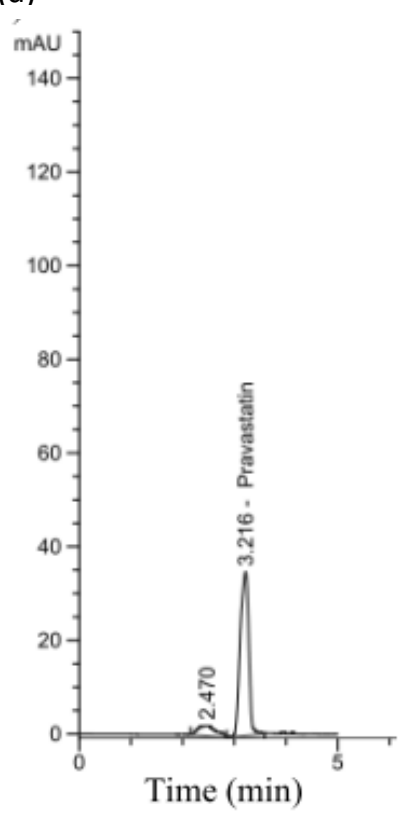

(b)

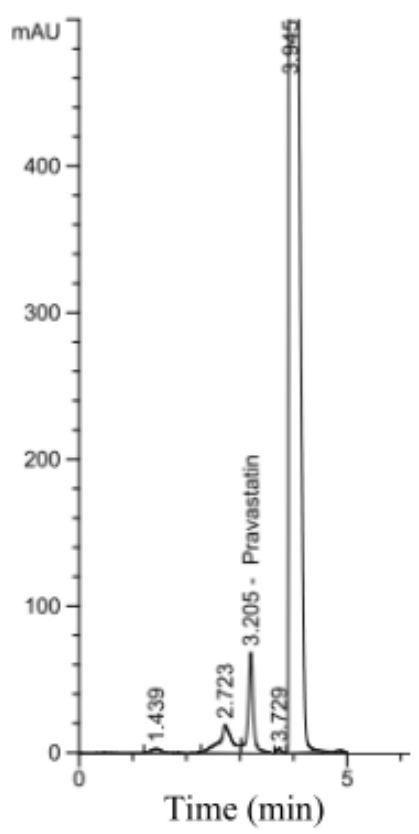

Fig. 1. High-performance liquid chromatograms: (a) Standard pravastatin;

(b) Ethyl acetate extract of isolate ESF20P.

\subsection{Identification of the Selected Strain to Species Level}

Microfungi have historically been identified based on macroscopic and microscopic properties. Examination of the features of the colonies and microscopic characteristics of the conidiophores are commonly used methods in fungal identification [15], [16]. Thirty-three fungal isolates examined in this study were characterized by filamentous growth on agar media. Table 3 shows the cultural and morphological properties of the best pravastatin producer selected in this study. Obtained results were clearly similar to the literature description of Penicillium janthinellum [16].

Table 3. Cultural and Morphological Characteristics of the Examined Isolate in Comparison with Those Reported in the Literature for Penicillium janthinellum

\begin{tabular}{lll}
\hline \hline Character & Isolate ESF20Pa & P. janthinellum $^{\mathrm{b}}$ \\
\hline & Cultural properties & \\
CD on CYA, mm & $26-30$ & $18-43$ \\
Conidial mass color & grayish green & grayish green \\
CRC & yellowish brown & yellowish brown \\
CD on MEA, mm & $45-50$ & $27-62$ \\
Conidial mass color & greenish gray & greenish gray \\
CRC & yellowish brown & dull yellow \\
& Morphological properties & \\
Conidiophore & monoverticillate & monoverticillate \\
Phialide length, $\mu \mathrm{m}$ & $9.66-11.92$ & $6.5-12.0$ \\
Phialide shape & ampulliform & ampulliform \\
\hline
\end{tabular}

abbervations were recorded after incubation at $25^{\circ} \mathrm{C}$ for 7 days. $\mathrm{CD}=$ colony diameter; $\mathrm{CRC}=$ colony reverse color.

bLiterature data were primarily adapted from Matsukuma et al. [16].

cAll measurements are presented as extremes. 
The strategy used here for molecular identification of the selected pravastatin-producing strain was to identify this fungal isolate by means of ribosomal DNA (rDNA) sequence comparison and assessment of the percentage of nucleotide similarity with reference sequence. Genomic DNA from the best pravastatin-producing ESF20P strain was extracted. The ITS1-5.8S-ITS2 region of the rDNA was amplified successfully producing a single PCR product of the desired length, approximately 550-600 base pairs [30]. The purified amplicon was double-stranded sequenced. The pairwise aligned sequence data from the examined fungal isolate were then compared with available sequences in the GenBank database using the BLASTN program. The section of rDNA sequenced from the selected isolate included 533 base pairs. The complete ITS1 region occupied nucleotides 8 to 180, the 5.8S rDNA gene from nucleotides 181 to 337 and ITS2 from nucleotides 338 to 507. The sequence of the obtained amplicon showed $98 \%$ identity to Penicillium janthinellum CBS 340.48 (accession number: GU981585).

Thus, based on the morphological characteristics described, and the ITS1-5.8S-ITS2 nucleotide sequence obtained, the best pravastatin-producing strain was identified as Penicillium janthinellum ESF20P. The sequence data from this study were deposited in the GenBank database under the accession number: JX456373. This fungal culture has been deposited at the Microbial Culture Collection of the Universiti Malaysia Pahang (UMPCC/F/040).

\subsection{Phylogeny}

A phylogenetic tree shows the evolutionary relationships among the strain selected in this study and a range of species whose sequence information was obtained from GenBank (Fig. 2). Bootstrap values calculated from 1000 replicates are given at the branches. Through the alignment and cladistic analysis of homologous nucleotide sequences, it was shown that the sequence of the P. janthinellum ESF20P strain fell in the same sub-clade as of the known P. janthinellum CBS 340.48 species with bootstrap value of $100 \%$. Of the species included in the phylogenetic analysis, P. citrinum CBS 122452 and P. janczewskii CBS 354.48 appeared to be closely related to $P$. janthinellum ESF20P strain. Moreover, Fig. 2 illustrates that $P$. brevicompactum CBS 257.29 and P. bialowiezense CBS 227.28 species formed a well-supported clade (99\% bootstrap support). P. chrysogenum CBS 306.48 and P. flavigenum CBS 419.89 formed separate sub-clade (82\%); whereas P. viridicatum CBS 390.48 and P. aurantiogriseum CBS 324.89 grouped together with 97\% bootstrap support.

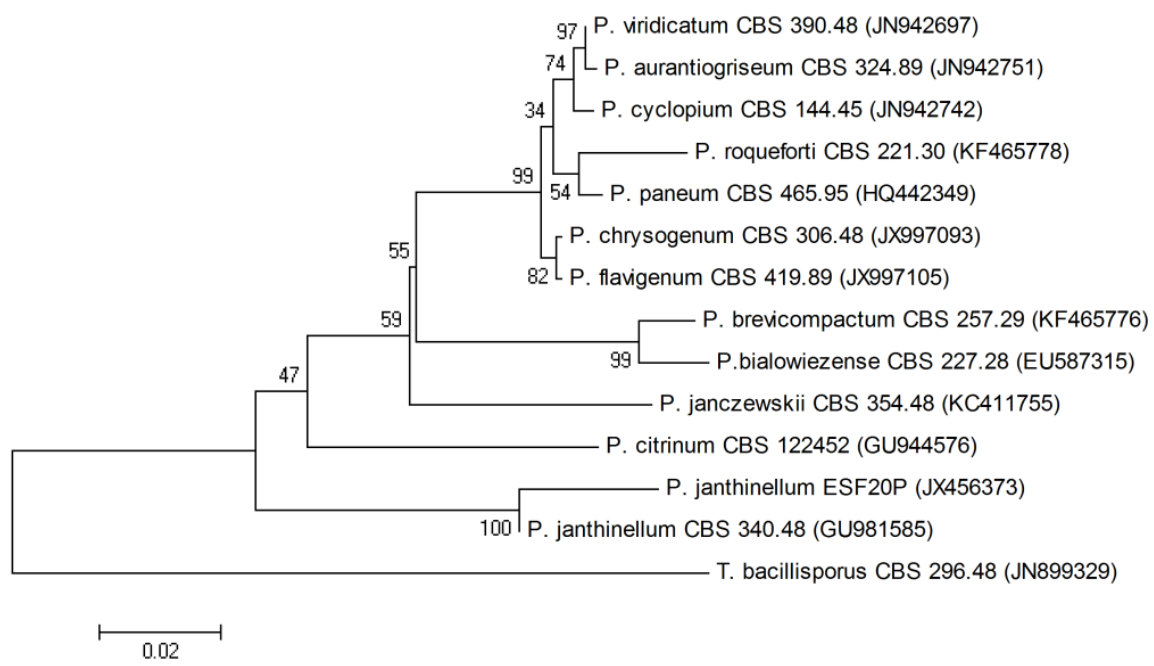

Fig. 2. Phylogenetic tree using the neighbor-joining method of representatives of Penicillium spp. inferred from analysis of the ITS region. 


\section{Conclusion}

The present study has identified a number of pravastatin-producing fungal strains obtained from oil palm plantation soils. All of them were able to synthesize pravastatin directly, although only isolate ESF20P did so in relatively high concentration. The most active pravastatin producer was identified to species level using standard morphological and molecular techniques, showing the highest sequence homology (98\%) with $P$. janthinellum species. In comparison with previous studies, this strain can already be considered as a novel fungal pravastatin producer of scientific interest. A further strain improvement program and optimization of the significant fermentation parameters, which are under investigation, may considerably enhance production of this fungal metabolite.

\section{Acknowledgment}

This work was supported by the Research and Management Center of the Universiti Malaysia Pahang under short-term Grants GRS090334 and RDU100319.

The author acknowledges Mr. Mohd Hafizi and Mr. Kelvin Tan Boon Seng from Yayasan Pahang Plantation Holding Sdn Bhd for providing soil samples.

\section{References}

[1] Pahan, K. (2006). Lipid-lowering drugs. Cellular and Molecular Life Sciences, 63(10), 1165-1178.

[2] Hebert, P. R., Gaziano, J. M., Chan, K. S., \& Hennekens, C. H. (1997). Cholesterol lowering with statin drugs, risk of stroke, and total mortality: An overview of randomized trials. The Journal of the American Medical Association, 278, 313-321.

[3] Demain, A. L. (1999). Pharmaceutically active secondary metabolites of microorganisms. Applied Microbiology and Biotechnology, 52, 455-463.

[4] Furberg, C. D. (1999). Natural statins and stroke risk. Circulation, 99, 185-188.

[5] Tobert, J. A. (1987). New developments in lipid-lowering therapy: The role of inhibitors of hydroxymethylglutaryl coenzyme. A reductase. Circulation, 76, 534-538.

[6] Chong, P. H., Seeger, J. D., \& Frankin, C. (2001). Clinically relevant differences between the statins: Implications for therapeutic selection. American Journal of Medicine, 111, 390-400.

[7] Barrios-González, J., \& Miranda, R. U. (2010). Biotechnological production and applications of statins. Applied Microbiology and Biotechnology, 85(4), 869-883.

[8] Hatanaka, T., Honda, S., Sasaki, S., Katayama, K., \& Koizumi, T. (1998). Pharmacokinetic and pharmacodynamic evaluation for tissue-selective inhibition of cholesterol synthesis by pravastatin. Journal of Pharmacokinetics and Biopharmaceutics, 26(3), 329-347.

[9] Ditschuneit, H. H., Kuhn, K., \& Ditschuneit, H. (1991). Comparison of different HMG-CoA reductase inhibitors. European Journal of Clinical Pharmacology, 40, 27-32.

[10] Pan, H. Y. (1991). Clinical pharmacology of pravastatin, a selective inhibitor of HMG-CoA reductase. European Journal of Clinical Pharmacology, 40, 15-18.

[11] Muniandy, M., Ahmed, O. H., Majid, N. M., \& Yusop, M. K. (2009). Effects of converting secondary forest to oil palm plantation on peat soil carbon and nitrogen and other soil chemical properties. American Journal of Environmental Sciences, 5(3), 406-412.

[12] Warcup, J. H. (1950). The soil-plate method for isolation of fungi from soil. Nature, 166, 117-118.

[13] Konya, A., Jekkel, A., Suto, J., \& Salat, J. (1998). Optimization of compactin fermentation. Journal of Industrial Microbiology and Biotechnology, 20, 150-152.

[14] Manzoni, M., Bergomi, S., Rollini, M., \& Cavazzoni, V. (1999). Production of statins by filamentous fungi. Biotechnology Letters, 21, 253-257. 
[15] Frisvad, J. C., \& Samson, R. A. (2004). Polyphasic taxonomy of Penicillium subgenus Penicillium. A guide to identification of the food and air-borne terverticillate penicillia and their mycotoxins. Studies in Mycology, 49, 1-173.

[16] Matsukuma, S., Nishizuka, Y., Watanabe, J., \& Okuda, T. (1994). Identification of Penicillium sp. NR 6564 and taxonomic notes on Penicillium janthinellum. Mycoscience, 35, 183-186.

[17] White, T. J., Burns, T., Lee, S., \& Taylor, J. W. (1990). Amplification and direct sequencing of fungal ribosomal RNA genes for phylogenetics. In M. A. Innis, D. H. Gelgard, J. J. Sninsky, \& T. J. White (Eds), PCR Protocols: A Guide to Methods and Applications (pp. 315-322). New York: Academic Press.

[18] Patino, B., Medina, A., Domenech, M., Gonzalez-Jaen, M. T., Jimenez, M., \& Vazquez, C. (2007). Polymerase chain reaction (PCR) identification of Penicillium brevicompactum, a grape contaminant and mycophenolic acid producer. Food Additives and Contaminants, 24(2), 165-172.

[19] Henry, P. C., Iwen, S., \& Hinrichs, H. (2000). Identification of Aspergillus species using internal transcribed spacer regions 1 and 2. Journal of Clinical Microbiology, 38, 510-515.

[20] Subramaniam, S. (1998). The Biology Workbench - a seamless database and analysis environment for the biologist. Proteins, 32, 1-2.

[21] Tamura, K., Dudley, J., Nei, M., \& Kumar, S. (2007). MEGA 4: Molecular evolutionary genetics analysis (MEGA) software version 4.0. Molecular Biology and Evolution, 24, 1596-1599.

[22] Carlile, M. J., Watkinson, S. C., \& Gooday, G. W. (2001). The Fungi. London: Academic Press.

[23] Kirk, J. L., Beaudette, L. A., Hart, M., Moutoglis, P., Klironomos, J. N., Lee, H., et al. (2004). Methods for studying soil microbial diversity. Journal of Microbiological Methods, 58, 169-188.

[24] Gunde-Cimerman, N., Friedrich, J., Cimerman, A., \& Benicki, N. (1993). Screening of fungi for the production of an inhibitor of HMG-CoA reductase: Production of mevinolin by the fungi of the genus Pleurotus. FEMS Microbiology Letters, 111, 203-206.

[25] Kumar, M. S., Kumar, P. M., Sarnaik, H. M., \& Sadhukhan, A. K. (2000). A rapid technique for screening of lovastatin-producing strains of Aspergillus terreus by agar plug and Neurospora crassa bioassay. Journal of Microbiological Methods, 40, 99-104.

[26] Chakravarti, R., \& Sahai, V. (2002). Optimization of compactin production in chemically-defined production medium by Penicillium citrinum using statistical methods. Process Biochemistry, 38, 481-486.

[27] Shindia, A. A. (1997). Mevinolin production by some fungi. Folia Microbiologica, 42(5), 477-480.

[28] Bazarra, W. A., Hamdy, M. K., \& Toledo, R. (1998). Bioreactor for continuous synthesis of compactin by Penicillium cyclopium. Journal of Industrial Microbiology and Biotechnology, 2, 192-202.

[29] Shaligram, S., Singh, S. K., Singhal, R. S., Szakacs, G., \& Pandey, A. (2008). Compactin production in solid-state fermentation using orthogonal array method by Penicillium brevicompactum. Biochemical Engineering Journal, 41, 295-300.

[30] Dean, T. R., Kohan, M., Betancourt, D., \& Menetrez, M. Y. (2006). A simple polymerase chain reaction sequencing analysis capable of identifying multiple medically relevant filamentous fungal species. Mycopathologia, 162, 265-271.

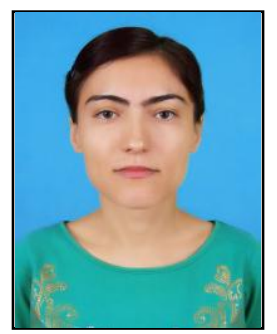

Emine Seydametova was born in Uzbekistan in 1980. She received her B.Sc. with honours in microbiology (2001) and the M.Sc. with honours in biochemistry (2003) from the National University of Uzbekistan, Tashkent, Uzbekistan; the Ph.D. in biotechnology from the Institute of Microbiology under Academy of Sciences of Uzbekistan (2009), Tashkent, Uzbekistan; the Ph.D. in bio-process engineering from the Universiti Malaysia Pahang (2013), Kuantan, Pahang State, Malaysia. 
Her work experiences are as follows: laboratory technician (2000-2001); senior laboratory technician (2002-2003); research assistant (2003); doctoral researcher (2003-2006); researcher (2006-2009) at the Institute of Microbiology under Academy of Sciences of Uzbekistan; doctoral researcher (2009-2013) and researcher at the Universiti Malaysia Pahang. She is an author of two monographs. Moreover, she has published several research papers in leading national and international journals, such as Proceedings of the Academy of Sciences of Uzbekistan, Acta of the National University of Uzbekistan, Microbiology, Applied Biochemistry and Microbiology, International Journal of Chemical Engineering and Applications. She has presented her research at many major national and international conferences, congresses, and symposiums. Her research interests are as follows: isolation and identification of microorganisms from different natural sources; screening of microbial cultures for biotechnologically valuable metabolites; strain improvement program; optimization of fermentation parameters using statistical tools; kinetic studies and scaling up; isolation, purification, and chemical structure identification of microbial metabolites.

Her memberships in professional societies are as follows: Young Scientists Board of the Institute of Microbiology under Academy of Sciences of Uzbekistan, Uzbekistan; Malaysian Natural Products Society (MNPS), Malaysia; Society for Applied Microbiology (SFAM), UK; Federation of European Microbiological Societies (FEMS), Netherlands; Asia-Pacific Chemical, Biological and Environmental Engineering Society (APCBEES), China. List of awards: Scholarship of the National University of Uzbekistan for undergraduate students (1999-2001); Scholarship granted by the President of Uzbekistan for master-students (2002-2003); Honorary Diploma for the best scientific lecture (2003); IREX Fellowship for young researchers from Uzbekistan (2003); World Concern Fellowship for talented youth from Uzbekistan (20032004); Doctoral Scholarship Scheme from the Government of Uzbekistan (2003-2006); Fellowship from the Government of India for young researchers from Uzbekistan (2008); Universiti Malaysia Pahang Doctoral Scholarship Scheme (2009-2012); TWAS-ARO Fellowship for a promising scientist from the developing country (2010); Fellowships from TWAS and Scientific Foundation of Lyon for a brilliant young researcher from the developing country (2011). She is a reviewer for International Journal of Bioscience, Biochemistry and Bioinformatics; International Journal of Chemical Engineering and Applications; International Journal of Environmental Science and Development and Current Microbiology Journal. 